\title{
Manejo respiratorio en atrofia muscular espinal: cambio de paradigma en la era de las nuevas terapias específicas
}

\section{Respiratory care in spinal muscular atrophy in the new therapeutic era}

\author{
María Angélica Palomino ${ }^{\mathrm{ab}, \mathrm{d}}$, Claudia Castiglioni ${ }^{\mathrm{a}, \mathrm{c}, \mathrm{e}}$ \\ aPrograma Trastornos Motores y Enfermedades Neuromusculares, Clínica Las Condes

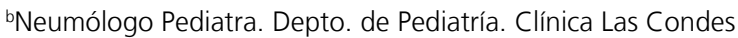 \\ `Neurólogo Pediatra. Depto. Neurología Pediátrica. Clínica Las Condes \\ dDepartamento de Pediatría Norte. Universidad de Chile \\ eDepartamento de Pediatría Sur. Universidad de Chile
}

Recibido el 10 de noviembre de 2018; aceptado el 12 de noviembre de 2018
Palabras clave: Atrofia muscular espinal;

Enfermedad neuromuscular; Enfermedad de Werdnig Hoffmann; Enfermedad pulmonar restrictiva; Ventilación no invasiva; Ventilación invasiva; Asistencia mecánica de la tos; Nusinersen 


\section{Abstract}

Spinal muscular atrophy (SMA) is the first inherited cause of mortality in infants, with four subtypes: SMA0 prenatal onset, SMA1 babies less than 3 months non sitters, SMA2 sitters and SMA3 walkers. Pneumonia and respiratory insufficiency are the most severe complications. Informed parental decisions are relevant. Respiratory management includes cough assistance, prevention of lung underdevelopment due to chest deformity, prompt treatment of respiratory infections, hypoventilation, swallow problems, gastro esophageal reflux and malnutrition. In view of the FDA and EMA approval of the nonsense oligonucleotides nusinersen, the first specific treatment for SMA and the future with gene therapy and others under development, we need to optimize preventive respiratory management with the new standard of care.

\section{Keywords:}

Spinal Muscular Atrophy;

Neuromuscular disease; Werdnig Hoffmann's Disease; Airway clearance; Mechanical cough assistance; Pneumonia; Respiratory failure; Restrictive lung disease; Non-invasive ventilation, Invasive ventilation; Nusinersen.

\section{Introducción}

La atrofia muscular espinal (AME) es una enfermedad neurodegenerativa de herencia autosómica recesiva que ocasiona muerte progresiva de las motoneuronas originadas en la médula espinal y región bulbar con la consecuente debilidad muscular. Es la primera causa genética de mortalidad en lactantes principalmente debido a complicaciones respiratorias. Su incidencia es de 1/10.000 nacimientos y su prevalencia es de 1-2/100.000 personas. Los pacientes con AME tipo 1 o enfermedad de Werdnig Hoffmann representan el $50 \%$ de todos los tipos de AME. La historia natural de la enfermedad en estos niños nos ha enseñado que fallecen antes de los 24 meses de edad por insuficiencia respiratoria, a menos que reciban cuidados clínicos preventivos; el pronóstico vital de estos pacientes se ha modificado significativamente con la incorporación de apoyos nutricionales y respiratorios consensuados por grupos de expertos en el mundo, creando consensos de estándar de manejo en pacientes con $\mathrm{AME}^{1-5}$. Un porcentaje creciente de estos niños vive más allá de los 2 años.

En la actualidad nos encontramos enfrentados a un cambio significativo en la manera como entendemos la enfermedad, gracias al vertiginoso desarrollo de diversas terapias específicas que están cambiando radicalmente lo que conocíamos como su historia natural ${ }^{6-8}$. Los tratamientos específicos modificadores de la enfermedad incorporan el desarrollo de nuevas moléculas y estrategias terapéuticas altamente específicas como el incremento de la proteína SMN a través de modificaciones del splicing del RNA mensajero o la terapia génica. Respaldado por ensayos clínicos multicéntricos de varios años, muchos de ellos de gran efectividad en los análisis provisorios, permitieron que en diciembre del año 2016 se aprobara por la FDA el uso de nusinersen (spinraza) de uso intratecal en pacientes con AME siendo la primera terapia específica para esta enfermedad $^{9-11}$. Recientemente en octubre del 2018 AveXis, Inc., una compañía de Novartis, solicitó oficialmente la aprobación por la FDA de AVXS-101 para pacientes AME tipo 1. Esta terapia reemplaza a través de una única dosis intravenosa, el gen de sobrevida de neurona motora SMN1 que esta ausente o mutado en pacientes con $\mathrm{AME}^{12}$. Es bajo esta perspectiva que hemos querido realizar esta actualización, para resaltar la importancia de los cuidados respiratorios en AME, ya no desde lo reactivo, sino desde lo preventivo, a la luz de los desafíos impuestos frente la emergencia de estas nuevas terapias; los pediatras deben aumentar los esfuerzos por perfeccionar las atenciones en salud de estos pacientes y optimizar sus condiciones globales a la espera de estos tratamientos específicos.

\section{Formas de presentación}

La severidad de las manifestaciones clínicas en AME es un continuo. Existe espectro amplio en relación al grado de debilidad muscular de extremidades y al compromiso respiratorio, con un desarrollo cognitivo normal. Se han definido cuatro subtipos principales en la población pediátrica. AME tipo 0 es de inicio en el período prenatal y al nacer los pacientes no presentan esfuerzo respiratorio, requieren ser ventilados y no presentan ninguna actividad motora, por lo que habitualmente fallecen en las primeras semanas de vida. AME tipo 1 se presenta en menores de 3 meses y son incapaces de sentarse. AME tipo 2 aparece en niños que se sientan, pero no logran caminar. La AME tipo 3 se diagnostica en niños que logran caminar de manera independiente ${ }^{7,8,13}$.

\section{Genética}

Esta enfermedad es consecuencia de una deleción o mutación homocigota del gen de sobrevida de la motoneurona (SMN1) ubicado en el brazo largo del cromo- 
soma 5. La región del cromosoma 5 donde esta asentado el gen $S M N 1$ es una región inestable que alberga una duplicación invertida única a los seres humanos de 500 kilobases donde se encuentra el gen SMN2, copia en espejo centromérica de SMN1. Ambos genes difieren solo en un cambio nucleotídico en la secuencia codificante en el exón 7, que afecta el splicing o empalme, provocando la exclusión del exón 7 en la transcripción de la proteína del gen SMN2. La proteína truncada originada de SMN2 entonces se degrada rápidamente en un $80 \%$ a $90 \%$ de las veces. El $10 \%$ de proteína SMN completa que produce la trascripción del gen SMN2 no alcanza a compensar la ausencia de proteína producida por la deleción de SMN1. Debido a que el gen SMN2 se encuentra en varias copias en el ser humano desde 1 hasta mas de 5, el gen SMN2 es un modificador de gravedad de la enfermedad existiendo una correlación inversa entre la severidad de la enfermedad y el número de copias de $S M N^{3,7,8}$.

\section{Compromiso respiratorio}

El compromiso respiratorio en la AME es de causa multifactorial con mecanismos relacionados entre sí. Los músculos respiratorios están comprometidos de forma variable, dependiendo del tipo de AME y del estado de la enfermedad: músculos inspiratorios, espiratorios, bulbares, con un diafragma proporcionalmente más fuerte. El desbalance entre la contractilidad diafragmática y la distensibilidad de la caja torácica favorece el desarrollo del tórax acampanado y la depresión esternal o pectus excavatum. Esto es especialmente importante en AME 1 y 2. En AME 1 la insuficiencia respiratoria es inevitable, en AME 2 es frecuente, especialmente frente a intercurrencias virales, e infrecuente en AME $3^{7,8,13}$. La debilidad de los musculos inspiratorios -intercostales- favorecen el desarrollo de atelectasias, la disminución de la distensibilidad torácica y por lo tanto de la capacidad vital $(\mathrm{CV})$. Aparece rigidez y fibrosis de tendones, ligamentos y articulaciones costales, con acortamiento de las fibras musculares. La respiración que es de tipo diafragmático, se hace paradojal, rápida, superficial y sin suspiros, lo que aumenta el trabajo respiratorio y la fatiga, el tiempo inspiratorio y la ventilación de espacio muerto. Los músculos abdominales e intercostales débiles reducen el reflejo de la tos. ${ }^{1,4,14} \mathrm{La}$ actividad diafragmática se hace insuficiente en posición supina, ocurriendo inicialmente durante el sueño. En AME 1 existe además compromiso bulbar, el que es variable en AME 2. Esto causa alteración de la deglución de saliva y alimentos, alteraciones respiratorias del sueño, que son de origen central, obstructivas o mixtas. Durante el sueño con movimientos oculares rápidos (REM), por la disminución del volumen corrien- te en pacientes con disfunción diafragmática severa aparece hipoventilación, eventos hipóxicos, retención de bicarbonato y depresión del centro respiratorio. En inspiración aparecen eventos obstructivos por la presión negativa que se ejerce sobre una faringe y laringe débil, produciendo el colapso de la vía aérea superior ${ }^{15}$. Como estos eventos aparecen inicialmente durante el sueño se recomienda investigarlos activamente ${ }^{1,4}$. La escoliosis es una complicación frecuente en pacientes con AME debido a la debilidad de la musculatura de tronco, abdominal, paravertebral y respiratoria. Esta aparece casi siempre en los pacientes con AME tipo 2, contribuyendo a la limitación respiratoria restrictiva y al desarrollo de atelectasias ${ }^{1,4,16,17}$. Si bien en años anteriores se debía esperar el término del crecimiento para someter a los pacientes a una artrodesis definitiva, hoy la tendencia es intervenir en forma más precoz a estos pacientes con barras crecedoras o magnéticas que permiten optimizar los problemas ventilatorios y el confort $^{3,18}$. La AME es una enfermedad neurodegenerativa con una progresión del deterioro lenta, sobre todo en pacientes con AME tipo 2 y 3; los pacientes presentan mesetas de estabilidad de años, con una disminución lenta de la capacidad vital y de otros parámetros. Parte de este deterioro se relaciona a la insuficiente ganancia de fuerza y masa muscular para alcanzar las demandas asociadas al crecimiento y desarrollo normal del niño ${ }^{1,4,14}$.

\section{Evaluación de la función respiratoria}

En lactantes o niños muy débiles la evaluación de la función respiratoria es inicialmente clínica. Se debe vigilar la efectividad de la tos, la frecuencia respiratoria, la respiración paradojal y el intercambio gaseoso ${ }^{4}$.

\section{Tos}

La tos se afecta precozmente y los factores que influyen en la eficacia de ella son la CV, la capacidad de insuflación máxima (CIM) y la presión inspiratoria máxima (PIMax), siendo menos importante la presión espiratoria máxima (PEMax). En niños mayores de 12 años se realiza a través del pico flujo de tos (PFT) con un flujómetro portátil o con el neumotacómetro utilizado para la realización de la espirometría. Valores de PFT menores a $270 \mathrm{~L} / \mathrm{min}$ predice un mayor riesgo de complicaciones respiratorias y si el PFT es menor a 160 la tos es inefectiva ${ }^{19,20}$.

\section{Función pulmonar}

La CV es un buen indicador de avance de la enfermedad y de necesidad de ventilación no invasiva (VNI). Se mide en posición erguida y en decúbito supino desde los 4 a 6 años; una diferencia de $20 \%$ evi- 
dencia la disfunción diafragmática. Una $\mathrm{CV}<1,1$ litro predice un riesgo mayor de complicaciones durante las infecciones respiratorias. En pacientes con escoliosis se recomienda la estimación de la talla por envergadura o longitud cubital. Es necesario considerar los valores de referencia utilizados en cada laboratorio de función pulmonar ${ }^{1,2,4}$.

\section{Fuerza de músculos respiratorios}

Su evaluación se realiza a través del PIMax y PEMax. El paciente realiza un esfuerzo inspiratorio máximo a partir de espiración completa y esfuerzo espiratorio máximo después de una inspiración completa; la maniobra de PIMax se realiza desde volumen residual, mientras que la de PEMax desde capacidad pulmonar total. Una PIMax disminuida refleja debilidad de los músculos inspiratorios, principalmente del diafragma y la PEMax de los músculos espiratorios, incluidos los abdominales. La PIMax es una maniobra simple, sin embargo requiere de coordinación entre el técnico y el paciente y requiere de aprendizaje ${ }^{18}$. Una técnica complementaria a la PIMax es la medición de la presión inspiratoria nasal máxima (SNIP o sniff nasal inspiratory pressure), que es la medición de la presión generada en la nariz al realizar una inhalación forzada desde capacidad residual funcional. No requiere de coordinación, no participa la musculatura facial y no se altera frente a problemas de la vía aérea superior ${ }^{21}$.

\section{Trastornos respiratorios del sueño}

La polisomnografía es esencial para la pesquiza de las apneas centrales, obstructivas o mixtas. Una alternativa de menor costo, especialmente para el seguimiento, es la poligrafía que permite realizar el estudio en la casa. ${ }^{4}$

\section{Manejo respiratorio}

La aproximación de un equipo multidisciplinario de neurólogo, pediatra neumólogo, fisiatra, kinesiólogo expertos en terapia respiratoria y motora, nutriólogo, cirujano de columna entre otros y la educación de los cuidadores y/o pacientes en esencial. Los padres deben ser informados y preparados en forma progresiva sobre los el objetivos de los tratamientos, para la toma de decisiones anticipadas ${ }^{3,4}$. El neumólogo debe involucrarse en el manejo proactivo inmediatamente después del diagnóstico. En AME 1 el objetivo es mejorar la calidad de vida. Con la disponibilidad en un futuro cercano de las nuevas terapias específicas este concepto cambiará. Las evaluaciones deben ser periódicas, mensuales en AME 1 y cada 3 a 6 meses en AME 2 y 3 , con el fin de adelantar los problemas que presentarán. Desde el punto de vista respiratorio esto incluye la ausencia del reflejo de tos y eliminación de secreciones respiratorias, la deformación de la caja torácica en una etapa crítica del desarrollo pulmonar, la hipoventilación del sueño y las infecciones respiratorias (tabla 1). Las atelectasias aumentarán aún más la debilidad muscular, con una recuperación que puede tomar varias semanas una vez resuelta la infección. Se debe detectar y tratar la alteración de la deglución y el reflujo gastroesofágico que favorece la aspiración pulmonar ${ }^{1-4}$. La desnutrición, frecuente en la formas más severas de AME puede aumentar la debilidad muscu$\operatorname{lar}^{22}$. La escoliosis progresiva, la distensión abdominal producida por fecalomas y la obesidad son factores que disminuyen la $\mathrm{CV}^{1,3,4}$. En adolescentes con AME 2 y 3 es frecuente observar obesidad que favorece los trastornos respiratorios del sueño ${ }^{4}$. No existe correlación directa entre la alteración pulmonar y la necesidad de asistencia ventilatoria. La evidencia existente se basa en series de casos y en consensos de expertos que se han ido modificando a la luz de las nuevas terapias ${ }^{4,23}$. Las vacunas necesarias incluyen neumococo y anualmente influenza. Se ha sugerido considerar en algunos casos el uso de anticuerpos monoclonales contra el virus respiratorio sincicial ${ }^{1,4}$.

\section{Tos y manejo de secreciones}

Los cuidadores se deben entrenar en la aspiración de secreciones, en la asistencia manual de la tos con ambú o idealmente con el asistente mecánico de la tos insufflator-exsufflator o Cough Assist (MI-E). Esto se debe realizar diariamente para evitar la formación de tapones mucosos y microatelectasias ${ }^{1,2,4}$. La expansión del tórax es necesaria para mantener su distensibilidad y promover el crecimiento pulmonar ${ }^{22}$. La asistencia mecánica de la tos a través de una interfase oronasal puede ser utilizada desde lactantes, ya sea en forma diaria para la expansión del tórax y durante las infecciones respiratorias, aumentándola si la saturación de oxígeno cae bajo $95 \% \%^{4,26,27}$. La cooperación se obtiene desde los 2 años, sin embargo, en menores de 12 meses es necesario ajustarlo a los esfuerzos inspiratorios y espiratorios. Las presiones efectivas deben aumentarse rápidamente desde $\pm 30 \mathrm{a} \pm 40-60 \mathrm{~cm} \mathrm{H}_{2} \mathrm{O}$ según tolerancia. El objetivo es lograr una expansión rápida y completa, y luego eliminar totalmente el aire del tórax. El uso del MI-E debería ser un estándar de manejo en AME 1,2,4. El glicopirolato puede ser útil para reducir las secreciones oro faríngeas y salivación excesiva, en dosis adecuadas, evitando que las secreciones se resequen ${ }^{4}$.

\section{Asistencia ventilatoria}

La ventilación mecánica no invasiva (VNI) debe iniciarse cuando hay evidencia de hipoventilación nocturna cuyos síntomas son despertares, cefalea matinal, 
Tabla 1. Manejo preventivo de las complicaciones respiratorias en atrofia muscular espinal

\begin{tabular}{|c|c|c|}
\hline Tipo & Evaluación & Tratamiento \\
\hline AME 1 & $\begin{array}{l}\text { - Evaluación cada } 1 \text { a } 3 \text { meses } \\
\text { - Examen físico: calidad de la tos, presencia de respiración } \\
\text { paradojal o diafragmática, taquipnea, taquicardia, tórax en } \\
\text { campana y/o pectus, diámetro torácico } \\
\text { - Síntomas de disfunción bulbar (atoros, disfagia, salivación) } \\
\text { - Saturación de oxígeno } \\
\text { - Evaluación clínica de reflujo gastroesofágico y trastorno de } \\
\text { la deglución } \\
\text { - Polisomnografía en caso de sospecha de trastornos respi- } \\
\text { ratorios del sueño: SAHOS, hipoventilación. } \mathrm{TCPCO}_{2} \text { si está } \\
\text { disponible en caso de sospecha de hiperventilación nocturna }\end{array}$ & $\begin{array}{l}\text { - Educación de los cuidadores } \\
\text { - Manejo de secreciones: aspiración oral y nasal, kinesiotera- } \\
\text { pia respiratoria. No usar mucolíticos. } \\
\text { - Manejo de la salivación excesiva: glicopirrolato en dosis } \\
\text { adecuadas para evitar sequedad de secreciones. No hay } \\
\text { consenso sobre el uso de toxina botulínica } \\
\text { - Estimulación diaria de la tos: manual con ambú o con el } \\
\text { asistente mecánico } \\
\text { - Evitar el pectus: insuflación diaria del tórax con ambú } \\
\text { - VNI con BIPAP: en pacientes sintomáticos o idealmente en } \\
\text { forma preventiva con respiración paradojal para evitar colap- } \\
\text { so pulmonar frente a infección respiratoria } \\
\text { - Prevención de infecciones respiratorias: educación y vacunas } \\
\text { neumococo e influenza anual en mayores de } 6 \text { meses. Con- } \\
\text { siderar uso de palivizumab en menores de } 2 \text { años. } \\
\text { - Medición de la saturación de oxígeno: si saturación baja de } \\
\text { 95\% obliga a manejo intensivo de la tos. } \\
\text { - Trastorno de deglución: gastrostomía precoz o sonda naso- } \\
\text { gástrica } \\
\text { - Tratamiento del reflujo gastroesofágico }\end{array}$ \\
\hline AME 2 & $\begin{array}{l}\text { - Evaluación cada } 3 \text { a } 6 \text { meses } \\
\text { - Examen físico: efectividad de la tos, mecánica respiratoria, } \\
\text { taquipnea, taquicardia, tórax en campana y/o pectus. } \\
\text { - Medición de saturación de oxígeno } \\
\text { - Espirometría sentado y acostado desde los } 4 \text { a } 6 \text { años, PFT, } \\
\text { PIM, PEM, SNIP (anual si CVF > } 60 \% \text { y cada } 6 \text { meses si es } \\
\text { < } 60 \% \text { ) } \\
\text { - Estudio de sueño frente a la sospecha de trastorno respira- } \\
\text { torio del sueño y si CV es menor } 60 \% \\
\text { - Evaluación clínica de reflujo gastroesofágico } \\
\text { - Radiografía de tórax al menos cada } 2 \text { años }\end{array}$ & $\begin{array}{l}\text { - Prevención de infecciones respiratorias: educación y vacunas } \\
\text { neumococo e influenza anual en mayores de } 6 \text { meses. Con- } \\
\text { siderar uso de palivizumab en menores de } 2 \text { años } \\
\text { - Manejo de secreciones: kinesioterapia, aspiración de secre- } \\
\text { ciones, estimulación de la tos. No usar mucolíticos } \\
\text { - VNI con BIPAP nocturna en pacientes sintomáticos y durante } \\
\text { las exacerbaciones respiratorias para acortar la evolución de } \\
\text { la enfermedad } \\
\text { - Identificar a pacientes con asma y tratarlos }\end{array}$ \\
\hline AME 3 & $\begin{array}{l}\text { - Evaluación cada } 6 \text { meses y en infecciones respiratorias } \\
\text { - Evaluación clínica de la tos o en infecciones respiratorias } \\
\text { - Espirometría sentado y acostado } \\
\text { - Estudio de sueño } \\
\text { - Evaluación clínica de reflujo gastroesofágico }\end{array}$ & $\begin{array}{l}\text { - Apoyo en exacerbaciones respiratorias } \\
\text { - Prevención de infecciones respiratorias: educación, vacunas } \\
\text { neumococo e influenza anual }\end{array}$ \\
\hline
\end{tabular}

ME: Atrofia Muscular Espinal. SAHOS: Sindrome apnea hiponea obstructiva del sueño. TCPCO : Anhidrido carbónico transcutaneo. PFT: pico flujo de la tos. PIM: presión inspiratoria máxima. PEM: presión espiratoria máxima. SNIP: presión inspiratoria nasal máxima. CV: Capacidad vital. VNI: ventilación no invasiva. BIPAP: presión positiva en la vía aérea bi-nivel.

fatiga, somnolencia diurna y dificultad en la concentración. Esto debería ser evaluado mediante una polisomnografía o poligrafía. Los gases matinales son de baja sensibilidad y molestos para el niño, sin embargo podría ser de utilidad la medición de $\mathrm{PCO}_{2}$ de forma no invasiva ${ }^{2,4}$. La VNI es necesaria además cuando existe dependencia para gatillar un flujo de aire adecuado, cuando hay descompensaciones con atelectasias que requieren la administración de oxígeno, para lograr una extubación exitosa, en anticipación a la descompensación por infección respiratoria, en el manejo perioperatorio de una gastrostomía o cirugía de escoliosis, en dependencia de ella o para dar de alta al domicilio con cuidados paliativos ${ }^{1,4,25}$. Los consensos internacionales sugieren que también debería iniciarse si existe tos inefectiva, pectus y tórax acampanado y/o respiración paradojal ${ }^{1,2,4}$. El ventilador portable debe ser siempre bi-nivel ${ }^{1,2,4}$. Las presiones inspiratorias inicialmente bajas, deben ser aumentadas rápidamente hasta lograr que desaparezca la respiración paradojal; esto ocurre generalmente sobre 12 o $14 \mathrm{~cm} \mathrm{H}_{2} \mathrm{O}$, aumentando a $16-20 \mathrm{~cm}$ de $\mathrm{H}_{2} \mathrm{O}$ según la edad del niños, manteniendo una span o presión de soporte-IPAP-EPAP- mayor a $10 \mathrm{~cm}$ de $\mathrm{H}_{2} \mathrm{O}$. Esto facilita la eliminación de $\mathrm{CO}_{2}$ y mantiene el tamaño de la caja torácica y pulmonar. El volumen corriente ideal para estos pacientes es alto y oscila entre 8 y $12 \mathrm{ml} / \mathrm{kg}$. El ventilador debe tener frecuencia respiratoria de respaldo y debe programarse una o dos respiraciones bajo la respiración espontánea en vigilia. La VNI a través de una interface nasal en AME 1 debe iniciarse precozmente. Para evitar la falta de tolerancia a la interface deben utilizarse las másca- 
ras nasales o almohadillas con materiales suaves para evitar las lesiones de la cara, considerando tener al menos dos tipos. La disfunción bulbar y la alteración de la deglución son contraindicaciones relativas de $\mathrm{VNI}^{2,4,26}$.

\section{Morbilidad asociada}

La nutrición óptima es indispensable. El índice de masa corporal objetivo en estos pacientes está entre percentil 25 y 50 . Se debe evitar la obesidad frecuente en AME 2 y $3^{22}$. En los casos más severos por disfunción bulbar con la debilidad de la lengua, dificultad para abrir la boca y pobre control de la cabeza aparecerá alteración de deglución o demoras excesivas en la alimentación, que obligan a planificar una gastrostomía precoz, asociada o no a cirugía de Nissen. Se debe buscar y tratar el reflujo gastroesofágico. Las complicaciones gastrointestinales también consideran la alteración de la motilidad intestinal que favorece la constipación ${ }^{4}$.

\section{Infecciones respiratorias}

Las neumonías y atelectasias son frecuentes y están generalmente relacionadas a las infecciones virales $y / o$ a eventos aspirativos. ${ }^{1,2,4}$ Las aproximaciones terapéuticas son cada vez menos controversiales. El desarrollo de obstrucción de la vía aérea por tapones mucosos $\mathrm{y}$ atelectasias por el aumento de secreciones favorece la hipoventilación alveolar e hipercapnia, con disminución del volumen corriente y el aumento de la frecuencia respiratoria. Si la saturación de oxígeno baja de $95 \%$, esto se debe a la hipoventilación o a la alteración de la relación ventilación-perfusión por aumento de secreciones, atelectasias o neumonía. La fatiga muscular aumenta, favoreciendo aún más las atelectasias que llevan a hospitalización, intubación endotraqueal $y$, en ocasiones, la muerte. Es por esta razón que cuando aparece la respiración paradojal será necesario el apoyo ventilatorio. Es importante monitorizar la saturación de oxígeno y mantenerla sobre $95 \%$, utilizando la tos asistida a fin de evitar hospitalizaciones. No se recomienda administrar oxígeno suplementario en la casa ya que esto puede aumentar la hipercapnia. Si no logran revertir la saturación de oxígeno con la estimulación de la tos será necesario tratamiento hospitalizado. La administración de antibióticos en estos pacientes tiene un umbral más bajo que en niños sin esta patología, si bien no hay evidencias de su utilidad. En aquellos usuarios de VNI nocturna será necesario aumentarla a las horas diurnas, con el fin de evitar la intubación endotraqueal y la ventilación mecánica invasiva. Si la intubación es inevitable, podría extubarse hacia la VNI continua con un span sobre $10 \mathrm{~cm} \mathrm{H}_{2} \mathrm{O}$, siempre y cuando la saturación de oxígeno esté sobre $94 \%$ en aire ambiental y la $\mathrm{pCO}_{2}$ sea normal, las secreciones sean escasas y no exista atelectasia, de acuerdo a lo propuesto por $\mathrm{Bach}^{27}$. Si el paciente aún requiere oxigenoterapia para mantener saturación arterial sobre 95\% debe intensificarse la kinesiterapia respiratoria y el uso del asistente de la tos. Se intentará la extubación a su interface habitual, pero en ocasiones será necesario utilizar transitoriamente una interface facial total. El protocolo de Bach incluye el uso del MI-E a través del tubo endotraqueal tan frecuente como sea necesario, con presiones +-25-+-30 cm de $\mathrm{H}_{2} \mathrm{O}$ hasta revertir la desaturación, extubación si está afebril, sin signos de infección bacteriana, sin requerimientos de oxígeno, con mejoría de la radiografía de tórax, sin depresores respiratorios, mínima necesidad de aspiración, idealmente menos de 1 a 2 cada $8 \mathrm{~h}$, y escasa coriza, monitorizar la saturación de oxígeno como guía de drenaje postural, kinesiterapia respiratoria y uso del MI-E para revertir cualquier desaturación debido a un tapón mucoso. Si se requiere reintubar, el protocolo se utiliza para un segundo intento de extubación. La kinesiterapia, el uso frecuente del asistente de la tos y la aspiración de secreciones nasofaríngeas debe mantenerse aun después de la extubación, planificando el alta una vez que la saturación esté sobre $95 \%$ por $24-48 \mathrm{~h}^{27}$.

\section{Aspectos éticos}

En pacientes con atrofia muscular de mayor gravedad como AME 1, luego del diagnóstico los padres luego de informarse de la enfermedad y junto al equipo tratante deben evaluar las alternativas terapéuticas: historia natural con intervención mínima, traqueotomía y ventilación mecánica invasiva crónica o soporte ventilatorio no invasivo acompañado de la asistencia mecánica de la tos. Un sicólogo experto dará contención emocional a los padres y al equipo médico, ayudando a la comprensión e integración de la información ${ }^{29-30}$. Estas decisiones se han visto alteradas con advenimiento de las nuevas terapias específicas. ${ }^{8}$

\section{Nuevas terapias}

La comunidad médica y científica ha sido testigo de mejoras clínicas notables en pacientes con AME a partir de dos nuevas estrategias de tratamiento para aumentar la proteína SMN: una de ellas mediante la modulación del splicing de los pre-ARNm de SMN2 utilizando oligonucleótidos antisentido (ASO) y la segunda a través de la transducción de motoneuronas con virus adeno asociados complementarios 9 (scAAV9) que son capaces de expresar el ADNc de $S M N 1^{8-12}$.

\section{Oligonucleótidos antisentido}

La primera de las terapias utiliza oligonucleótidos antisentido para mejorar la inclusión del exón $7 \mathrm{du}-$ rante el splicing de los ARNm de SMN2. Los ASO son 
ácidos nucleicos de una sola hebra diseñados para hibridar con su secuencia de ARN diana mediante el acoplamiento de bases. La identificación de un elemento regulador negativo en el intrón 7, el silenciador de empalme intrónico N1 (ISS-N1) fue clave para comprender los mecanismos reguladores del splicing del exón 7 de $S M N 2^{31}$. Nusinersen es un oligonucleótido diseñado para hibridizar con ISS-N1 y de esta manera alterar por impedimento estérico la unión del represor del splicing, incrementando la inclusión del exón 7 y permitiendo la expresión de una proteína SMN completa. Como los ASO no atraviesan la barrera hematoencefálica la administración de esta molécula requiere la vía intratecal. La capacidad de nusinersen de distribuirse eficientemente en el sistema nervioso central fue demostrada por trabajos en roedores y primates en el que se observó una eficiente distribución y penetración de la molécula a lo largo de la médula espinal y motoneuronas $^{32}$. El protocolo de administración de Nusinersen contempla una dosis de carga repartida en dos meses y terapia de mantención cada 4 meses. Los ensayos clínicos demostraron altos niveles de seguridad y eficacia de este producto lo que llevó a la aprobación para la comercialización e indicación como tratamiento en AME tipo 1, 2 y $3^{9-11}$. Cabe destacar el ensayo clínico NURTURE entre los diversos ensayos clínicos diseñados con nusinersen. En este ensayo aun en curso, fase 2 abierto (Nurture, NCT02386553) se analizaron los efectos de nusinersen en 25 bebés presintomáticos con AME tipo1 o 2. El análisis provisorio de octubre del año 2017 estableció que de los 9 niños que habían completado el análisis al día 365, ninguno había fallecido o requerido intervención respiratoria, todos podían sentarse, cinco podían gatear, tres podían pararse solos, cinco podían caminar con apoyo y dos podían caminar independientes ${ }^{33}$. Estos resultados confirman que el inicio precoz de tratamiento con nusinersen proporciona mayores beneficios clínicos que en etapas mas avanzadas y sintomáticas de la enfermedad.

\section{Terapia génica}

En los últimos años varios grupos de investigación han demostrado con éxito la transfección del gen SMN1 a través de la inyección endovenosa sistémica de un virus adeno-asociado (scAAV) ${ }^{34}$. Los AAV son una familia de virus no patógeno, pequeños, de ADN de una sola hebra, sin envoltorio y que requieren la coinfección de un adenovirus "ayudante" para la replicación. Varias razones inclinaron a los investigadores a utilizarlos como vectores virales de terapia génica. Una de estas es su potencial para transducir neuronas, las otras son la mínima integración al genoma del huésped que reduce la preocupación por la oncogénesis, y la baja respuesta inflamatoria e inmune que presentan, lo que mejora su tolerancia en comparación con otros vectores virales. El reemplazo de gen SMN1 por un vector viral scAVV9, transportador del gen humano demostró ser exitoso en restaurar los niveles de proteína SMN. Este vector inyectado por vía endovenosa en dosis única logra traspasar la barrera hematoencefálica e infecta eficazmente las motoneuronas logrando de esta manera restaurar niveles más adecuados de proteína SMN. Después de pasar con éxito los ensayos preclínicos el año 2014, se dio inicio al primer ensayo clínico en humanos para la administración sistémica de AAV9 ${ }^{35}$.

El ensayo de transferencia de génica de Avexis AAV9-SMN fue un ensayo piloto abierto de 15 recién nacidos con AME menores de seis meses con la intención de estudiar la dosis, la seguridad de la dosis única de scAAV9-SMN intravenosa y proporcionar una visión temprana de la eficacia según la duración de la supervivencia y la función motriz. Tres pacientes toleraron bien una dosis baja de genomas viral $(6,7 \times 1.013$ $\mathrm{vg} / \mathrm{kg}$ de peso corporal), lo que otorgó la aprobación para una dosificación mas alta a los siguientes 12 niños $(2 \times 1.014 \mathrm{vg} / \mathrm{kg}$ de peso corporal). El ensayo sigue en curso, pero ya se ha comunicado que todos los lactantes actualmente sobre los 20 meses de edad están vivos y 11 de los 12 en el grupo de dosis más alta tenían ganancias significativas en la función motora nunca antes observada para estos pacientes. Once niños lograron sentarse de forma independiente, 9 rodar y 2 caminar independientemente ${ }^{12}$.

\section{Otras estrategias terapéuticas}

Estas incluyen terapia con células madres, moléculas neuroprotectoras y compuestos que aumentan la fuerza muscular ${ }^{8}$. El desarrollo de intervenciones terapéuticas futuras dependerá de una comprensión más acabada de otros factores modificadores del fenotipo de la enfermedad, aparte del ya conocido gen SMN2. Los estudios preclínicos, realizados en el modelo de ratón "delta 7" portador del transgen SMN2 humano, así como los resultados obtenidos en los ensayos NURTURE de Biogen para pacientes pre-sintomáticos y el ensayo de vector viral AVV9 de avexis para lactantes con AME1, nos indica que mientras más precoz es el reemplazo de la proteína SMN normalmente disminuida en este cuadro, mayores son los efectos en el grado de reversión del fenotipo. Esto sugirió la existencia de ventanas terapéuticas estrechas que han debido ser consideradas en los diseños y criterios de inclusión de los ensayos clínicos actualmente en curso. También ha acelerado la implementación del tamizaje neonatal de AME en varios países.

Los potenciales efectos del aumento de la proteína SMN en etapas más tardías, en fenotipos clínicos menos severos de la enfermedad, donde cambios menores en la fuerza muscular pueden modificar en forma sig- 
nificativa funciones que mejoran la vida de los pacientes, se encuentra aún en etapa de estudio. Hoy existen más de 17 ensayos clínicos en AME en diferentes etapas preclínicas y clínicas ${ }^{8}$.

\section{Conclusiones}

Estamos siendo testigos de un cambio de paradigma en el cuidado de los pacientes con AME. Ellos están ya accediendo o lo harán en un futuro a terapias específicas, por lo tanto la historia natural de la enfermedad cambiará si son intervenidos en forma precoz. Para que las nuevas terapias alcancen el mayor efecto en los pacientes es indispensable la implementación de un manejo multidisciplinario proactivo y preventivo de las numerosas complicaciones médicas de esta enfermedad progresiva. La reciente actualización de los cuidados en AME, orientan sobre el manejo respiratorio, nutricional, ortopédico, el uso de suplementos nutricionales, manejo de condiciones agudas y quirúrgicas en estos pacientes ${ }^{1,4}$. Los pediatras debemos estar atentos a estos cambios, adaptándonos a este tiempo "intermedio" mientras nos organizamos como país para que los niños con AME accedan a un diagnóstico pre- coz, ojalá con pesquisa neonatal, y a estos tratamientos específicos de alto costo ${ }^{36}$. Mientras tanto nuestra labor es optimizar el diagnóstico precoz y avanzar hacia un manejo preventivo, evitando las complicaciones que deteriorarán aun más la calidad de vida. Los pilares del manejo respiratorio son la estimulación de la tos, el tratar la hipoventilación nocturna y manejar oportunamente las infecciones respiratorias, el trastorno de deglución, el reflujo gastroesofágico y la malnutrición. El uso precoz de presión positiva y la estimulación de la tos favorecen el desarrollo adecuado de la caja torácica lo que permite el crecimiento pulmonar en los primeros años de la vida que son críticos para el desarrollo pulmonar. Dado que la morbilidad y mortalidad está dada fundamentalmente por las complicaciones respiratorias, es que debemos estar atentos a estos cambios para así optimizar los cuidados no invasivos y proactivos desde el momento del diagnóstico. Las consideraciones éticas aun no están resueltas en esta patología, es por esta razón que los padres deben tomar decisiones bien informadas.

\section{Conflicto de intereses}

Los autores declaran no tener conflicto de intereses.

\section{Referencias}

1. Wang $\mathrm{CH}$, Finkel RS, Bertini ES, et al. Standard of Care. Consensus statement for standard of care in spinal muscular atrophy. J Child Neurol. 2007;(8):102749 .

2. Sansone, Racca F, Ottonello G, et al. on behalf of the Italian SMA Family Association, 1st Italian SMA Family Association Consensus Meeting:, Neuromuscular Disorders 2015; 979-89.

3. Mercuri E, Finkel RS, Muntoni F, et al. SMA Care Group. Diagnosis and management of spinal muscular atrophy: Part 1: Recommendations for diagnosis, rehabilitation, orthopedic and nutritional care. Neuromuscul Disord 2018;28(2):103-15

4. Finkel RS, Mercuri E, Meyer OH, et al. SMA Care group. Diagnosis and management of spinal muscular atrophy: Part 2: Pulmonary and acute care; medications, supplements and immunizations; other organ systems; and ethics. Neuromuscul Disord 2018,28(3):197-207.

5. Ministerio de Salud. Guía Clínica Cuidados respiratorios en personas con Atrofia Muscular Espinal, Santiago: Minsal, 2014.
6. Oskoui M, Levy G, Garland CJ, et al. The changing natural history of spinal muscular atrophy type 1 . Neurology. 2007;69(20):1931-6.

7. Mercuri E, Bertini E, Iannaccone ST. Childhood spinal muscular atrophy: controversies and challenges. Lancet Neurol 2012;11(5):443-52.

8. Tizzano E. La atrofia muscular espinal en el nuevo escenario terapéutico. Rev Med Clin Condes 2018;29(5) 512-20.

9. Chiriboga CA, Swoboda KJ, Darras BT, et al. Results from a phase 1 study of nusinersen (ISIS-SMN (Rx)) in children with spinal muscular atrophy. Neurology, 86 (2016), pp. 890-97.

10. Finkel RS, Mercuri E, Darras BT, et al. Nusinersen versus sham control in infantile-onset spinal muscular atrophy. N Engl J Med. 2017;377:1723-32.

11. Mercuri E, Darras BT, Chiriboga CA, et al. CHERISH Study Group. Nusinersen versus Sham Control in Later-Onset Spinal Muscular Atrophy. N Engl J Med. 2018;378(7):625-35.

12. Mendell JR, Al-Zaidy S, Shell R, et al. Single-dose gene-replacement therapy for spinal muscular atrophy. N Engl J Med 2017; 377:1713-22.

13. Castiglioni C, Levican J, Rodillo E, et al. Atrofia muscular espinal: Caracterización clínica, electrofisiológica y molecular de
26 pacientes. Rev Med Chile 2011; 139: 197-204.

14. Schroth MK. Special Considerations in the Respiratory Management of Spinal Muscular Atrophy. Pediatrics 2009;123:S245-9.

15. Bersanini C, Khirani S, Ramirez A, et al. Nocturnal hypoxaemia and hypercapnia in children with neuromuscular disorders. Eur Respir J 2012;39:1206-12.

16. Ching S, Wong $\mathrm{Y}$, Hui J, Wong $\mathrm{H}$, Ong $\mathrm{H}$. Pulmonary function and scoliosis in children with spinal muscular atrophy types II and III. J Paediatr Child Health. 2003; 39(9).

17. Khirani S, Bersanini C, Aubertin G, Bachy M, Vialle R, Fauroux B. Non-invasive positive pressure ventilation to facilitate the post-operative respiratory outcome of spine surgery in neuromuscular children. Eur Spine J. 2014;23(4):S406-11.

18. Lorenz HM, Badwan B, Hecker MM, et al. Magnetically Controlled Devices Parallel to the Spine in Children with Spinal Muscular Atrophy. JB JS Open Access. 2017;2(4):e0036.

19. Torres-Castro R, Monge G, Vera R, Puppo H, Cespedes J, Vilaro J. Estrategias terapeuticas para aumentar la eficacia de la tos en pacientes con enfermedades neuromusculares. Rev Med Chil 2014;142(2):238-45. 
20. Bianchi C, Baiardi P. Cough peak flows: standard values for children and adolescents. Am J Phys Med Rehabil 2008;87(6):461-7.

21. Martínez-Llores J, Ausín P, Roig A, et al. Presión inspiratoria nasal: ¿una alternativa para la evaluación de la fuerza muscular inspiratoria? Arch Bronconeumol 2011;47:169-75.

22. Davis RH, Godshall BJ, Seffrood E, et al. Nutritional practices at a glance: spinal muscular atrophy type I nutrition survey findings. J Child Neurol. 2014;29:1467-72.

23. Benditt J. Initiating Noninvasive Management of Respiratory Insufficiency in Neuromuscular Disease. Pediatrics 2009;123:S236-8.

24. Bach JR. Prevention of precuts excavated for children with spinal muscular atrophy type I. Am J Phys Med Rehabil 2003;82:815-9.

25. Khirani S, Bersanini C, Aubertin G, Bachy M, Vialle R, Fauroux B. Non-invasive positive pressure ventilation to facilitate the post-operative respiratory outcome of spine surgery in neuromuscular children. Eur Spine J 2014; 23 (Suppl. 4):S406-11.
26. Bach JR, Gonçalves MR, Hon A, et al. Changing Trends in the Management of End-Stage Neuromuscular Respiratory Muscle Failure. Recommendations of an International Consensus. Am J Phys Med Rehabil. 2012;91:1-11.

27. Bach J. The use of mechanical ventilation is appropriate in children with genetically proven spinal muscular atrophy type 1 : the motion for. Paediatric Respiratory Reviews 2008;9:45-50.

28. Ryan MM, Kilham H, Jacobe S, Tobin B, Isaacs D. Spinal muscular atrophy type 1: is long-term mechanical ventilation ethical? J. Paediatr. Child Health. 2007;43:237-42.

29. Lillo s. El proceso de comunicar y acompañar a los padres y al paciente frente al diagnóstico de discapacidad. Rev Med Clin Condes 2014;25(2):372-9.

30. Palomino MA, Castiglioni C. Atrofia Muscular Espinal: manejo respiratorio en la perspectiva de los recientes avances terapeuticos. Rev Med Clin Condes 2017; 28(1):119-30.

31. Singh NK, Singh NN, Androphy EJ, Singh RN. Splicing of a critical exon of human
Survival Motor Neuron is regulated by a unique silencer element located in the last intron. Mol Cell Biol. 2006; 26(4): 133346.

32. Passini MA, Bu J, Richards AM, Kinnecom C, et al. Antisense oligonucleotides delivered to the mouse CNS ameliorate symptoms of severe spinal muscular atrophy. Sci Transl Med. 2011; 3(72):72ra18.

33. Chiriboga CA. Nusinersen for the treatment of spinal muscular atrophy. Expert Rev Neurother. 2017;17(10):95562.

34. Foust KD, Wang X, McGovern VL, et al. Rescue of the spinal muscular atrophy phenotype in a mouse model by early postnatal delivery of SMN. Nat Biotechnol. 2010 Mar; 28(3):271-4

35. Duque SI, Arnold WD, Odermatt P, et al. A large animal model of spinal muscular atrophy and correction of phenotype. Ann Neurol. 2015;77(3):399-414.

36. Faravelli I, Nizzardo M, Comi GP, Corti S. Spinal muscle atrphy, recent therapeutic advances for an old challenge. Nat Rev Neurol 2015; 351-9. 